\title{
The Effect of Treadmill Training Applied Simultaneously with Action Observation on Walking Ability in Chronic Stroke Patients
}

\author{
Yo-han Song ${ }^{1}$, Hyun-min Lee ${ }^{2}$ \\ 'Department of Rehabilitation Science, Graduated School of Honam University; ${ }^{2}$ Department of Physical Therapy, College of Health Science, Honam \\ University, Gwangju, Korea
}

Purpose: To investigate the effect of treadmill training applied simultaneously with gait related action observation on walking ability in chronic stroke patients.

Methods: Sixteen chronic stroke patients participated in this study. Participants were randomly allocated into either the treadmill applied simultaneously with action observation training group (TAG) or treadmill applied simultaneously with landscape observation training group (TLG). The participants in both group underwent treadmill training for four weeks (a total of twelve minute, once a day, three times weekly for a four week period). All participants were measured to gait speed (10 m walking test, $10 \mathrm{MWT}$ ), gait endurance (6 minute walk distance, 6 MWD), dynamic gait index (DGI). In order to assure the statistical significance of the results, we used for SPSS 15.0 for windows. The Wilcoxon signed ranks test was used to compare pre-test and post-test result, and the Mann-Whitney U-test was employed for comparison between groups.

Results: The 10 MWT, 6 MWD, DGI was significantly different between the TAG and the TLG group.

Conclusion: According the results of this study, treadmill applied simultaneously with action observation (TAG) is effective intervention for improvement of walking ability in chronic stroke patient.

Keywords: Action observation training, Treadmill training, Walking ability, Stroke

\section{INTRODUCTION}

Stroke is a prevalent neurologic disease that causes physical functional disorders and social disadvantages. ${ }^{1}$ The disabilities caused by stroke vary depending on the severity and region of the damage; however, the symptoms generally include motor and sensory paralysis, cognitive impairment, and visual impairment. ${ }^{2}$ The pathological characteristics of a stroke include muscle weakness due to paresis of the musculoskeletal system, abnormal movement patterns, and balance impairment. ${ }^{3,4}$ These lead to motor control disorders and, eventually, limitations in functional activities, such as walking, that are necessary to stroke patients for their daily activities at home or in the community. ${ }^{5-7}$

The independent walking function of stroke patients requires the

Received May 20, 2016 Revised Jun 23, 2016

Accepted Jun 27, 2016

Corresponding author Hyun-min Lee

E-mail leehm@honam.ac.kr ability to maintain balance during walking. ${ }^{8-11}$ Sufficient walking speed for various environmental and social situations and endurance to walk a certain distance are also important factors for leading an independent life. ${ }^{12-14}$ Recent approaches to overcoming such walking abilities differ from the existing neurophysiological and neurodevelopmental approaches in that they are focusing on the functional purposes, and they are bringing many changes to the therapeutic approaches on stroke patients..$^{15}$

Task-oriented training has shown to be more effective than the traditional approach in improving the functional activity of stroke patients. Repetitive task-oriented training improves the control of movements in daily activities or nerve roots, and recovers the motor skills. ${ }^{16,17}$ Treadmill training is one of the task-oriented training methods that involve repetitive walking practices, and its effective-

Copylight (C2016 The Korea Society of Physical Therapy

This is an Open Access article distribute under the terms of the Creative Commons Attribution Non-commercial License (Http:// creativecommons.org/license/by-nc/4.o.) which permits unrestricted non-commercial use, distribution, and reproduction in any medium, provided the original work is properly cited. 
ness has been proven in many previous studies. ${ }^{18-20}$ Other studies have reported that treadmill training is effective in improving the walking speed and endurance of stroke patients. ${ }^{21}$ It also shows better results compared to other methods, such as neurodevelopmental treatment or proprioceptive neuromuscular facilitation. ${ }^{22}$

Stroke patients with limited motor ability have a harder time participating in physical therapies. For this reason, they have limited exposure to experience-based neuroplastic input that is necessary for recovery. ${ }^{23}$ In order to overcome such limitations, recent evidence supported the effectiveness of action observation training during the post-stroke relearning process of functional tasks. ${ }^{24,25}$ Action observation training is used to improve the motor skills of a patient with motor disorders through the activation of the mirror neuron system. ${ }^{26,27}$ Other studies have also supported the effectiveness of action observation training for the improvement of walking ability and balance ability in stroke patients. ${ }^{28-30}$

When healthy subjects were observed while walking and crossing over obstacles on a treadmill, the anterior tibialis and soleus muscles showed the same reflex control as crossing over actual obstacles. This was reported as a form of spinal cord excitation that corrects the prepared actions within the action pattern ranges of near future, and modulates the reflex adaptations and estimated movements during action observation. ${ }^{31,32}$ When action observation was applied while walking on a treadmill, the orders from the upper motor neurons are controlled by the observed actions, and they do not inhibit the lower neurons. This control reacts largely to the changes caused by the mirror properties of the pyramidal tract neurons, and reflects the actions estimated from the action observations. The reports also suggested that reactions to changes have to offer the same stimulations, such as task-specific reactions or reflexes, as observing actual actions of crossing over obstacles, and that when the actions were observed while walking, the same spinal cord excitation corrected the movements. ${ }^{31-33}$ However, most studies on action observation are followed by physical training while imagining and copying the said actions. There are only a few studies that approach the action observation and physical training of stroke patients at the same time. In this study, we aimed to investigate the effects of action observations and treadmill training applied at the same time on the walking ability of stroke patients.

\section{METHODS}

\section{Subjects}

This study included 16 inpatients of C Rehabilitation Hospital, which was located in Gwangju. They have been diagnosed with post-stroke hemiparesis. Medical records of the included patients were checked in order to verify the eligibility according to the following criteria.

For inclusion in this study, subjects had to meet the following criteria: six months or more had passed after a stroke was diagnose; over 24 points in score of K-MMSE; ability to walk over $10 \mathrm{~m}$ without assistance; ability to train on a treadmill for 20 minutes; without the presence of other neurological or orthopedic damages; and without optical or general sensory disorders.

All participants were sufficiently informed on the details of the study, and they voluntarily agreed to participate. The study protocol was reviewed and approved by the Institutional Review Board. The purpose and method of the study were explained before data collection, and written agreements were obtained from every participant. All 16 participants signed a written agreement before participating in this study.

The 16 stroke patients were randomly allocated to two different groups. The TAG $(n=8)$ underwent treadmill training while watching a video related to walking. Meanwhile, the TLG $(n=8)$ underwent the same training, but watched a video of a scenery. The training sessions took place 3 times a week for 4 weeks, thereby amounting to a total of 12 sessions. Both groups were evaluated before and after the 4-week training by using functional assessment tools, such as 10-m walking speed test (10 MWT), 6-minute walking distance (6 MWD), and dynamic gait index (DGI) (Figure 1).

Ethical approval was obtained to commence this study from the Biomedical Ethics Committee of Honam University. The approval number was 1041223-201601-HR-003.

\section{Experimental methods}

\section{1) Measurement}

\section{(1) $10 \mathrm{MWT}$}

10 MWT is a very useful method for measuring the walking speed of chronic stroke patients with a high inter-rater and intra-rater reliability $(r=0.95-0.96) .{ }^{34}$ The subjects were instructed to walk a distance of $14 \mathrm{~m}$ both in a comfortable gait and a quicker gait. Then, 


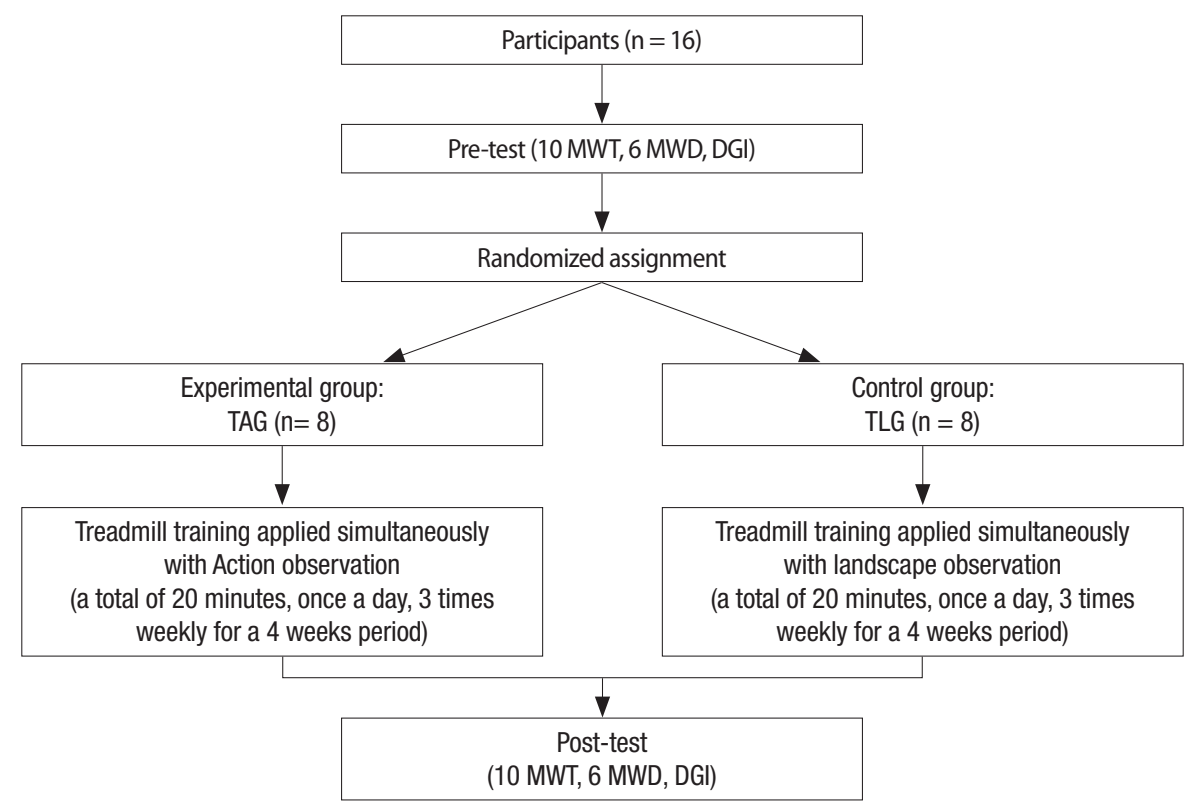

Figure 1. The study flowchart.

10 MWT: 10-min walk test, 6 MWD: 6 minute walking distance, and DGI: dynamic gait index.

the time duration for the middle $10 \mathrm{~m}$ was measured (the $2 \mathrm{~m}$ at the beginning and end are excluded for acceleration and deceleration). The test was repeated 3 times and the average duration (s) was selected for the result.

\section{(2) 6MWD}

The start and end points of a $30 \mathrm{~m}$ distance were marked on an indoor flat surface, and the participants were instructed to walk between the points as many times as possible for 6 minutes. An objective assessor informed the patient of the remaining time each minute. The result was measured by recording the total walking distance from the start to the end in meters. Six MWD has a high reliability of $r=0.91 .^{35}$

\section{(3) DGI}

DGI is a standardized assessment tool that evaluates the balance while walking of patients or elderly subjects with damaged balance ability. It can also evaluate changes in the gait as reactions to walking tasks, and it has a high reliability of $r=0.96 .{ }^{36}$ DGI consists of 8 walking tasks that include walking on a flat surface, walking at various speeds, walking with the head turned vertically or horizontally, walking through or around obstacles, and walking on stairs. Each task is graded 0-3 points based on the performance, and the total score may vary between 0 and 24 . Subjects with scores over 22 are
Table 1. Gait related action observation video
1. Treadmill walking video
2. Indoor walking video
3. Outdoor walking video
4. Ascent walking video
5. Downhill walking video
6. Avoid obstacle video
7. Skipping obstacle video
8. Uneven surface walking video

considered safe while walking, and scores under 19 may be considered as "elderly with risk of fall".

\section{Intervention}

The TAG watched a video of walking motions while training on a treadmill for 20 minutes, and the TLG underwent the training while watching a video unrelated to walking. All subjects started the training by walking on the treadmill at their chosen speed. After each training session, the treadmill training speed was increased by $10 \%{ }^{22}$ A study investigator stood beside or behind the participant during the training in order to prevent any unexpected falls. The video used in the TAG showed walking at $0.8 \mathrm{~m} / \mathrm{s}$ (speed sufficient for independent activity in various environments) and 1.1-1.5 m/s (speed sufficient for normal social life and crossing the street with a traffic light) which was shot from the front, side, and back. The action observation video of TAG included 8 tasks of walking (Table 1) (treadmill walking 
video, indoor walking video, outdoor walking video, ascent walking video, downhill walking video, avoid obstacle video, skipping obstacle video, uneven surface walking video). ${ }^{28,29}$ The video was 20 minutes long and shown 12 times during 4 weeks (20 minutes per session, 3 times a week). The TLG was shown a video with shots unrelated to walking, such as the scenery of mountains, fields, and beaches. This video was also shown 12 times during 4 weeks. The study investigator gave instructions such as: "Please look at the person in the video while walking." or "Please notice how the legs in the video move passing over the obstacle." in order to make sure that the participants are focused on the screen during treadmill training.

\section{Statistical analysis}

The statistical analysis of the data obtained was performed by using SPSS for Windows Ver. 15.0. The general characteristics of the subjects were analyzed by using descriptive statistics, and the differences in age and disease period (months) between the action observation treadmill training group and the landscape observation treadmill training group were analyzed through the use of an independent t-test. The normal distribution of the data was tested by using the Shapiro-Wilk test. The results from evaluations before and after the intervention and posttest results did not show a normal distribution; therefore, the Wilcoxon signed-rank test was used to investigate the difference between before and after the intervention. The results before and after the training were compared by using the difference between the values in the Mann-Whitney U-test. The $\mathrm{p}$-value was set at 0.05 .

\section{RESULTS}

\section{General characteristics of study objects}

A total of sixteen subjects, 14 men and 2 women, participated in this study. The average age was 56.25 in the TAG and 59.75 in the TLG (Table 2).

\section{Changes before and after the experiment between groups, changes within group}

The results of the comparison are shown in Table 3, 4. The 10 MWT of both groups showed a significant difference in the results taken

Table 2. General characteristics of the participants

\begin{tabular}{llccc}
\hline & & TAG $(\mathrm{n}=8)$ & TLG $(\mathrm{n}=8)$ & $\mathrm{p}$ \\
\hline Gender & Male & 7 & 7 & \\
\multirow{2}{*}{ Hemiplegic side } & Female & 1 & 1 & \\
\multirow{2}{*}{ Stroke type } & Right & 5 & 4 & \\
& Left & 3 & 4 & \\
Time since stroke (month) & Hemorrhage & 3 & 4 & \\
Age (years) & Infarction & 5 & 4 & \\
& & $32.25 \pm 26.92$ & $41.12 \pm 29.62$ & 0.587 \\
\hline
\end{tabular}

Mean \pm SD.

TAG: Treadmill training applied simultaneously with action observation, TLG: Treadmill training applied simultaneously with landscape observation.

Table 3. The comparison of variances within in each group

\begin{tabular}{|c|c|c|c|c|c|c|c|c|}
\hline \multirow{2}{*}{ Variable } & \multicolumn{4}{|c|}{ TAG } & \multicolumn{4}{|c|}{ TLG } \\
\hline & pre-test & post-test & z & $p$ & pre-test & post-test & z & $p$ \\
\hline $10 \mathrm{MWT}$ & $19.35 \pm 4.5$ & $13.61 \pm 3.26$ & -2.521 & $0.012^{*}$ & $18.80 \pm 4.35$ & $15.96 \pm 3.33$ & -2.521 & $0.02^{*}$ \\
\hline $6 \mathrm{MWD}$ & $212.25 \pm 82.94$ & $245.25 \pm 82.48$ & -2.524 & $0.012^{*}$ & $211.5 \pm 59.26$ & $231.62 \pm 63.66$ & -2.524 & $0.013^{*}$ \\
\hline DGI & $17.13 \pm 4.05$ & $20.50 \pm 3.16$ & -2.536 & $0.011^{*}$ & $15.87 \pm 2.64$ & $16.87 \pm 3.22$ & -2.271 & $0.023^{*}$ \\
\hline
\end{tabular}

TAG: Treadmill training applied simultaneously with action observation, TLG: Treadmill training applied simultaneously with landscape observation. ${ }^{*} p<0.05$.

Table 4. The comparison of variances between each group

\begin{tabular}{|c|c|c|c|c|c|}
\hline Variable & & $\mathrm{TAG}$ & TLG & z & $p$ \\
\hline \multirow[t]{2}{*}{ 10MWT } & Pre-test & $19.35 \pm 4.5$ & $18.8 \pm 4.35$ & -0.105 & 0.916 \\
\hline & Post-test & $13.61 \pm 3.26$ & $15.96 \pm 3.33$ & -2.521 & $0.012^{*}$ \\
\hline \multirow[t]{2}{*}{ 6MWD } & Pre-test & $212.25 \pm 82.94$ & $211.5 \pm 59.26$ & .000 & 1.00 \\
\hline & Post-test & $245.25 \pm 82.48$ & $231.62 \pm 63.66$ & -1.841 & 0.065 \\
\hline \multirow[t]{2}{*}{ DGI } & Pre-test & $17.13 \pm 4.05$ & $15.87 \pm 2.64$ & -0.532 & 0.595 \\
\hline & Post-test & $20.5 \pm 3.16$ & $16.87 \pm 3.22$ & -3.12 & $0.002^{* *}$ \\
\hline
\end{tabular}

TAG: Treadmill training applied simultaneously with action observation, TLG: Treadmill training applied simultaneously with landscape observation. ${ }^{*} p<0.05 ;{ }^{* *} p<0.005$. 
after the training, as compared to those taken before the intervention. The TAG showed a more prominently significant difference, as compared to the TLG. Although both groups showed a significant difference in the post-training $6 \mathrm{MWD}$ results, there was no significant difference between the groups. DGI showed a significant difference in the post-training results, and the TAG also showed a significant difference in the comparison between the groups.

\section{DISCUSSION}

This study aimed to investigate the effects of action observation and treadmill training applied at the same time on the walking ability of stroke patients. Sixteen stroke patients were included in the study and underwent 20 minutes training sessions 3 times a week for 4 weeks. Eight subjects were shown a video that included clips of walking on a treadmill, on a flat surface indoors and outdoors, on a slanted surface, around and over an obstacle, and on a lawn. The other 8 subjects were shown a video with shots unrelated to walking, such as the scenery of mountains, fields, and beaches. After 4 weeks of training, the walking ability of the participants was assessed by using functional assessment tools, such as 10 MWT, 6 MWD, and DGI.

The 10 MWT results showed an improvement of the TAG from $19.35 \pm 4.5$ seconds to $13.61 \pm 3.26$ seconds post-training. The TLG also showed improvement from $18.8 \pm 4.35$ seconds to $15.96 \pm 3.33$ seconds. The treadmill training is an effective method to improve the walking speed of stroke patients. ${ }^{37}$ Pohl et al. ${ }^{22}$ divided the stroke patients into 3 groups, namely, regular treadmill training group, treadmill training group in which the speed was gradually increased $10 \%$, and traditional walking training group, and compared the walking ability of each group. The study reported that only the group with gradually increased speed showed a post-intervention improvement from $0.66 \pm 0.42 \mathrm{~m} / \mathrm{s}$ to $0.97 \pm 0.64 \mathrm{~m} / \mathrm{s}$. In this study, the speed was gradually increased in both groups, which may explain the improvement of speed in both groups. The results that showed a significant difference in the TAG may suggest that simultaneously showing videos of faster walking $(0.8 \mathrm{~m} / \mathrm{s}$ and $1.1-1.5 \mathrm{~m} / \mathrm{s})$ may have provided a repetitive learning of the qualitative (normal movements) and quantitative (normal walking speed) aspects of walking, thereby improving the walking speed in comparison to the TLG.
The 6 MWD results showed significant post-intervention improvement from $212.25 \pm 82.94 \mathrm{~m}$ to $245.25 \pm 82.48 \mathrm{~m}$ and $211.5 \pm$ $59.26 \mathrm{~m}$ to $231.62 \pm 63.66 \mathrm{~m}$ in TAG and TLG, respectively. The comparison between the groups showed that there was no significant difference between them. In the study of Bang et al..$^{28} 10$-minute action observation training and 30-minute physical training were conducted, and as a result a significant difference was shown in 6 MWD between groups. However, in this study, it is thought that there was no significant difference between groups due to the 20-minute of short intervention time of the treadmill applied simultaneously with action observation. In a previous study, the walking distance that a subject can walk on the treadmill was compared with the walking distance on the ground, and the result revealed that treadmill training increased the walking distance that a subject can walk for a short time. These study results mean that the movement of lower limbs becomes larger during gait of the subjects due to the treadmill belt continuously moving, and the short-time walking distance more increases as active efforts are required depending on the speed of treadmill. ${ }^{38,39}$ In this study, since the treadmill was applied in both groups, there was a significant difference in 6 MWD before and after intervention.

The DGI scores showed improvement in both groups. The TAG group showed an increase from $17.13 \pm 4.05$ to $20.5 \pm 3.16$, while the TLG group showed an increase from $15.87 \pm 2.64$ to $16.87 \pm 3.22$. Park et al. ${ }^{29}$ reported an increase of DGI score from $12.09 \pm 5.15$ to $16.18 \pm 5.17$ in the action observation followed by physical training group. This result can be explained by the fact that the participants observed various states of walking motions that are easily accessed during daily activities, and then practiced walking under task-specific situations. In this study, the test group showed a more prominently significant difference in item number 6 (crossing over an obstacle) and item number 7 (walking around an obstacle). This improvement may be due to the fact that the motions of such items were included in the video, and performing the treadmill training while simultaneously observing various states of walking provided the subjects with learning both the qualitative and quantitative aspects of walking, thereby causing the subjects to walk while estimating future motions.

In this study, we investigated the effect of treadmill training with simultaneous action observation on the walking ability of a chronic stroke patient. The results of this study revealed that the TAG 
showed better improvement in walking ability, as compared to the TLG. However, there are a few limitations. First of all, the number of patients included in this study $(n=16)$ is too small to generalize the results to all stroke patients. Another limitation may be that the exact mechanism of the mirror neuron system and spinal reflex has not been fully discovered yet. Lastly, there are only a few existing studies that applied action observation and physical training at the same time, thereby making it difficult to conduct a proper comparison. In the future, clinical investigators should focus on studies to provide a standard frame of physical training with simultaneous action observation.

In this study, the walking abilities were evaluated by using functional assessment tools, such as $10 \mathrm{MWT}$ time, 6MWT distance, and DGI score. As a result, both groups showed a significant difference before and after the training results, which may be explained by the exercise effects of the treadmill training. The TAG showed a significant improvement in 10 MWT time and DGI score, as compared to the TLG. This suggests that walking on a treadmill while watching a video about walking provides a qualitative and quantitative learning of walking to stroke patients, and that the simultaneous action observation causes the patients to walk while estimating future motions. In conclusion, TAG may be an effective intervention for improving the walking ability of a chronic stroke patient.

\section{REFERENCES}

1. Eich H, Mach H, Werner C et al. Aerobic treadmill plus Bobath walking training improves walking in subacute stroke: a randomized controlled trial. Clin Rehabil. 2004;18(6):640-51.

2. Ozdemir F, Birtane M, Tabatabaei R et al. Cognitive evaluation and functional outcome after stroke. Am J Phys Med Rehabil. 2001;80(6): 410-5.

3. Hong SI, Bang DH, Shin WS. Effects of side walking training with elasticBand on gait and balance of stroke. J Kor Phys Ther. 2014;26(5):372-8.

4. Yang Dj, Park SK, Kang JI et al. Effects of game based weight-bearing training on lower extremity muscle activation and balance in stroke patients. J Kor Phys Ther. 2015;27(4):264-9.

5. Chen CL, Chen HC, Tang SF et al. Gait performance with compensatory adaptations in stroke patients with different degrees of motor recovery. Am J Phys Med Rehabil. 2003;82(12):925-35.

6. Sharp SA, Brouwer BJ. Isokinetic strength training of the hemiparetic knee: effects on function and spasticity. Archives of physical medicine and rehabilitation. 1997;78(11):1231-6.

7. Werner C, Bardeleben A, Mauritz KH et al. Treadmill training with partial body weight support and physiotherapy in stroke patients: a prelimi- nary comparison. Eur J Neurol. 2002;9(6):639-44.

8. Lee KS, Choe HS, Lee J-H. Influence of visual feedback training on the balance and walking in stroke patients. J Kor Phys Ther. 2015;27(6): 40712.

9. Ham SC, Lim CG. The effects of robot-assisted gait training with visual feedback on gait, balance and balance confidence in chronic stroke patients. J Kor Phys Ther. 2016;28(2):71-6.

10. Shin YI, Yang SH, Kim JY. Clinical feasibility of wearable robot orthosis on gait and balance ability for stroke rehabilitation: a case study. J Kor Phys Ther. 2015;27(2):124-7.

11. Yu Kh, Jeon HS. The effects of dual-task gait training on gait performance under cognitive tasks in chronic stroke. J Kor Phys Ther. 2015;27(5):364-8.

12. Lerner-Frankiel M, Vargas S, Brown M et al. Functional community ambulation: what are your criteria. Clin Manag Phys Ther. 1986;6(2):125.

13. Lord SE, Rochester L. Measurement of community ambulation after stroke current status and future developments. Stroke. 2005;36(7):145761.

14. Olney SJ, Monga TN, Costigan PA. Mechanical energy of walking of stroke patients. Arch Phys Med Rehabil. 1986;67(2):92-8.

15. Shumway-Cook A, Woollacott M. Aging and postural control. Motor Control: Theory and Practical Applications. 2nd ed. Baltimore, Williams \& Wilkins, 2001:222-47.

16. Carr JH, Shepherd RB. Stroke rehabilitation: guidelines for exercise and training to optimize motor skill. Oxford, Butterworth-Heinemann Medical, 2003.

17. Dobkin BH. Training and exercise to drive poststroke recovery. Nat Clin Pract Neurol. 2008;4(2):76-85.

18. Ada L, Dean CM, Vargas Jet al. Mechanically assisted walking with body weight support results in more independent walking than assisted overground walking in non-ambulatory patients early after stroke: a systematic review. J Physiother. 2010;56(3):153-61.

19. Kesar TM, Reisman DS, Perumal R et al. Combined effects of fast treadmill walking and functional electrical stimulation on post-stroke gait. Gait Posture. 2011;33(2):309-13.

20. Kim SY. Effect of treadmill training on walking velocity and gait endurance in patients with chronic hemiplegia. J Kor Phys Ther. 2004;16(2): 44-53.

21. Kim JJ, Rho MH, Goo BO et al. The effect of speed-dependent with body weight supported treadmill training on the ambulation of stroke. J Kor Phys Ther. 2005;17(3):339-50.

22. Pohl M, Mehrholz J, Ritschel C et al. Speed-dependent treadmill training in ambulatory hemiparetic stroke patients a randomized controlled trial. Stroke. 2002;33(2):553-8.

23. Garrison KA, Winstein CJ, Aziz-Zadeh L. The mirror neuron system: a neural substrate for methods in stroke rehabilitation. Neurorehabilitation and neural repair. 2010;24(5):404-12.

24. Ertelt D, Small S, Solodkin A et al. Action observation has a positive impact on rehabilitation of motor deficits after stroke. Neuroimage. 2007;36(Suppl 2): T164-73.

25. Kim JH, Lee BH. Action observation training for functional activities after stroke: a pilot randomized controlled trial. NeuroRehabilitation. 2013;33(4):565-74. 
26. Léonard G, Tremblay F. Corticomotor facilitation associated with observation, imagery and imitation of hand actions: a comparative study in young and old adults. Expl Brain Res. 2007;177(2):167-75.

27. Rizzolatti G, Fogassi L, Gallese V. Neurophysiological mechanisms underlying the understanding and imitation of action. Nat Rev Neurosci. 2001;2(9):661-70.

28. Bang DH, Shin WS, Kim SY et al. The effects of action observational training on walking ability in chronic stroke patients: a double-blind randomized controlled trial. Clin Rehabil. 2013;27(12):1118-25.

29. Park HR, Kim JM, Lee MK et al. Clinical feasibility of action observation training for walking function of patients with post-stroke hemiparesis: a randomized controlled trial. Clin Rehabil. 2014;28(8):794-803.

30. Kim JC, Lee HM. The effect of action observation training on sit to walk with chronic stroke patients. J Kor Phys Ther. 2015;27(6):413-8.

31. Behrendt F, de Lussanet MH, Wagner H. Observing a movement correction during walking affects evoked responses but not unperturbed walking. PloS One. 2014;9(8):e104981.

32. Behrendt F, Wagner H, de Lussanet MH. Phase-dependent reflex modulation in tibialis anterior during passive viewing of walking. Acta Psychol (Amst). 2013;142(3):343-8.
33. Takahashi M, Kamibayashi K, Nakajima T et al. Changes in corticospinal excitability during observation of walking in humans. Neuroreport. 2008;19(7):727-31.

34. van Loo M, Moseley A, Bosman J et al. Test-re-test reliability of walking speed, step length and step width measurement after traumatic brain injury: a pilot study. Brain Inj. 2004;18(10):1041-8.

35. Mossberg KA. Reliability of a timed walk test in persons with acquired brain injury. Am J Phys Med Rehabil. 2003;82(5):385-90.

36. Jonsdottir J, Cattaneo D. Reliability and validity of the dynamic gait index in persons with chronic stroke. Arch Phys Med Rehabil. 2007; 88(11):1410-5.

37. Sullivan KJ, Knowlton BJ, Dobkin BH. Step training with body weight support: effect of treadmill speed and practice paradigms on poststroke locomotor recovery. Arch Phys Med Rehabil. 2002;83(5):683-91.

38. Cahalin LP, Mathier MA, Semigran MJ et al. The six-minute walk test predicts peak oxygen uptake and survival in patients with advanced heart failure. Chest. 1996;110(2):325-32.

39. Sohn R, Choi H, Son J et al. The comparison of overground walking and treadmill walking according to the walking speed: motion analysis and energy consumption. J Biomed Eng Res. 2009;30(3):226-32. 